\title{
Nouvelles lignes directrices pour l'expertise rhumatologique
}

\section{Jörg Jeger}

Dr méd., responsable du groupe de travail Médecine des assurances, la Société Suisse de Rhumatologie, MEDAS, membre de la FMH

Partout dans le monde - et la Suisse n'est pas épargnée -, les affections psychiques et de l'appareil locomoteur entraînent le plus souvent des handicaps et des pertes de revenus. ${ }^{1}$ Les affections rhumatismales causent non seulement une grande souffrance, mais aussi des coûts considérables pour le diagnostic, le traitement et l'indemnisation des pertes de revenus. La Société Suisse de Rhumatologie est consciente de l'importance de cette réalité sur le plan médico-social. En tant que seconde société de discipline médicale de Suisse, la Société Suisse de Rhumatologie a rédigé en 2007 des lignes directrices pour l'évaluation de personnes souffrant d'affections rhumatismales.

La Communauté de travail des sociétés de discipline médicale scientifique allemande AWMF décrit l'importance des lignes directrices de la manière suivante: «Les lignes directrices des sociétés de discipline médicale scientifique sont des aides développées de manière systématique destinées à aider les médecins à prendre des décisions dans des situations spécifiques. Elles reposent sur des connaissances scientifiques actuelles et des procédures éprouvées dans la pratique, et garantissent davantage de sécurité dans le domaine de la médecine, tout en ayant à tenir compte également d'aspects économiques. Les lignes directrices ne sont pas juridiquement contraignantes pour les médecins et de ce fait, elles n'engagent ni ne dégagent les médecins de leur responsabilité..»²

La capacité de travail est un terme juridique, et elle est au bout du compte laissée à l'appréciation de l'instance chargée d'appliquer le droit. En l'occurrence, cette dernière a besoin d'une base médicale sérieuse. Les lignes directrices peuvent contribuer d'une manière essentielle à améliorer la qualité de l'expertise. Lors de l'évaluation de la performance d'une personne, l'expert dispose encore cependant d'une certaine latitude. Il est impossible d'éliminer totalement un certain écart entre les évaluations. C'est pourquoi l'on confie l'expertise à des «experts», notamment des médecins, qui apportent leurs connaissances scientifiques et leur expérience professionnelle issues du contexte clinique.
Ce sont précisément ces connaissances et cette expérience que l'instance chargée d'appliquer le droit ne possède pas elle-même et qu'elle trouve chez l'expert.

Pour pouvoir atteindre un haut niveau de transparence, d'exactitude et de répartition équitable, droit et médecine doivent collaborer étroitement. Ainsi, le Tribunal fédéral a consigné, dans sa nouvelle version de la jurisprudence sur les affections psychosomatiques (ATF 141 V 281 du 3.6.2015), ce qui suit: «Les sociétés de discipline médicale compétentes communiqueront dans des lignes directrices l'état actuel des connaissances à l'attention du cabinet d'expertise.» Le Tribunal fédéral vient de rendre une décision de principe obéissant à une compréhension moderne de la maladie et du handicap lorsqu'il écrit: «L'incapacité de travail découle pour ainsi dire du solde de toutes les dépenses et de toutes les ressources essentielles.»

Cette décision a donné à la Société Suisse de Rhumatologie une double occasion de remanier les lignes directrices datant de 2007: la première édition avait déjà 8 ans et la nouvelle version de la jurisprudence du Tribunal fédéral a, notamment dans le domaine de l'évaluation des personnes souffrant de douleurs chroniques, une influence directe sur les expertises rhumatologiques et orthopédiques.

Le groupe de travail Médecine des assurances a remanié les lignes directrices pour le compte de la SSR. Le groupe de travail se composait des personnes suivantes:

- Dr méd. Walter Kaiser, Thalwil, président de la SSR de 2012 à 2016

- PD Dr méd. Andreas Klipstein, Zurich, président de la SIM, membre du comité de la SSMPR

- Dr méd. Isabelle Gabellon, Vevey, présidente de l'ARPEM, membre du comité de la SSR

- Prof. Dr méd. Peter Villiger, Berne, membre du comité de la SIM, président de la SSR de 2004 à 2008

- Dr méd. Pius Brühlmann, Zurich

- Dr méd. Dieter Frey, Bâle

- Dr méd. Jörg Jeger, Lucerne (direction) 
Comme c'était déjà le cas pour la première édition 2007, le juge fédéral Prof. Dr iur. U. Meyer, du Tribunal fédéral de Lucerne, nous a cette fois encore conseillés sur le plan juridique, nous l'en remercions sincèrement.

Lors de la mise à jour, environ les $2 / 3$ du texte de l'édition 2007 ont été repris. Ces «nouvelles» lignes directrices devraient constituer un support, notamment pour les «apprenants» (médecins en formation postgraduée et médecins spécialistes qui se forment désormais à l'expertise). La Classification of Functioning, Disability and Health (ICF) a solidement ancré nos références. Ce qui nous paraissait important était la mise en évidence des questions de l'ATF $141 \mathrm{~V}$ 281, dans la mesure où l'expertise rhumatologique en dépend: détermination des diagnostics basée sur des critères, estimation de la gravité de l'affection, preuve du handicap, contrôle de cohérence, évaluation des motifs d'exclusion. De plus, le glossaire a été considérablement étendu. L'interface entre le droit et la médecine implique que le médecin expert entre souvent en contact avec des notions de droit qui ne lui sont pas familières, et en dehors du contexte clinique. Il faut également mentionner la collaboration avec l'Association Romande des Praticiens en Expertises Médicale (ARPEM), la communauté d'intérêts de la Swiss Insurance Medicine (SIM) et de la Société Suisse de Médecine Physique et de Réadaptation.
La nouvelle version des lignes directrices a été approuvée par le comité de la Société Suisse de Rhumatologie le 2.6.2016 et présentée aux membres lors de l'Assemblée annuelle à Interlaken le 25.8.2016. Elle a été publiée en septembre 2016 sur le site Web de la SSR (www. rheuma-net.ch) en français et en allemand, et peut être consultée et téléchargée de façon illimitée.

La Société Suisse d'Orthopédie et de Traumatologie (swiss orthopaedics) a repris ces lignes directrices et y a apporté certaines adaptations nécessairement liées au domaine de spécialité. Les lignes directrices de la société de discipline médicale en orthopédie ont été mises en ligne le 15.2.2017 sur le site Web (www.swiss orthopaedics.ch).

On dispose maintenant de lignes directrices sur l'expertise médicale d'affections et de séquelles d'accidents sur l'appareil locomoteur dont de grandes parties sont uniformes.

Les lignes directrices sur papier ne garantissent encore à elles seules aucune amélioration de la procédure d'expertise. Une formation des utilisateurs et une prise en compte conséquente du quotidien des experts sont importantes. De premiers ateliers ont déjà été réalisés. 